Ophthalmologica 1994; 208:347-348

\title{
Subject Index Vol. 208,1994
}

Acanthamoeba keratitis 321

Accommodative strabismus surgery 71

Actinomyces 314

Active force 201

Acute retinal necrosis 49

Adenoma 172

Air tonometry 115

- $\quad$ pulse 309Albino rats 98Amblyopia 214Anaesthetics 309Angioid streaks 239

Anterior chamber inoculation 325

Anterior-chamber-associated immune deviation 325

Antiglaucoma agents 278

Antioxidant enzymes 5

Apparatus 98

Applanation tonometry 115, 309

Arteritic anterior ischemic optic neuropathy 336

Atropine sulfate 207

Attenuated total reflection 17

Automated perimetry 10

Avellino corneal dystrophy 198

B-scan 192

Bacterial contamination 77

Bare-sclera technique 247

Bear track spots 175

Behçet's disease 210

Benign epithelioma 172

Bilateral keratitis 321

ß-Blockers 32

Blow-out fracture 284

Branch retinal vein occlusion 29

Canaliculitis 314 Canaliculotomy 314 Carteolol 32 Cataract 5,254

extraction 77 Cefmenoxime 262 Cell-mediated immune response 325 Cellular immunity 49

Chlamydia trachomatis 295 Choroidal blush 185

melanoma 65

neovascularization 185 Ciliary body 298 Colchicine 210

Color Doppler imaging 304

- $\quad$ vision tests 23

Columnar-type cell 267

Computed tomography 201

Computer 98 
Cone mechanism 136

Congenital grouped pigmentation 175

Conjunctiva 41

Conjunctival papilloma 46

Conjunctivitis 161

Conservative treatment 314

Contact lens 214,321

Corneal astigmatism 131,250

endothelial permeability 179

endothelium 289

epithelium 37

healing 37

organ culture 262

perforation 112

sensitivity 216 Corticosteroid 210 CTscan 329 CT-20 tonometer 115 Cyclophotocoagulation 122

Cyclosporin 210 Cytokeratin 289 Cytology 295

Cytotoxic T lymphocytes 105, 325

Dark adaptation 23

Decongestant eyedrops 243

Deformation 17

Degeneration 270

Delayed choroidal filling 185

Delayed-type hypersensitivity 325

Demyelinating disorder 226

Diabetes 179

Differential vector 250

Digitalis-induced colour vision deficiencies

259 Direct immunofluorescence 295 Diurnal variation 110 Drusen, optic nerve head 239 Dry eye 216 Dysthyroid myopathy 201

Echographic measurement 192 Echography 239 Electroretinography 98

sensitivity 220 Emory mouse 5 Endothelial cell toxicity 262

pump function 179 Enzymatic degradation 278 Epidermal growth factor 37

Erythema infectiosum 161 Ethyl alcohol 17 Excimer laser 198 Experimental model 157

trauma 270 Extended wear 214 Exudative retinal detachment 29 Eye patch 214

tissues 5 Eyelid 54

Failed retinal detachment surgery 207 Familial exudative vitreoretinopathy 233 Family study 23

Farnsworth-Munsell 100-hue test 259 Female 163 Fish-hook injury 112 Fluorescein angiography 168, 239 Fluorophotometry 179 5-Fluorouracil 318 Foreign bodies 192 Fungal keratitis 333

Fungi 314

Gamma knife 119 Giant cell arteritis 336 Glaucoma(s) 32, 254, 304, 309 Glutathione peroxidase 5 Glycoprotein C 157 Glycosaminoglycans 1,278 Goldmann tonometer 115

Hepatic carcinoma 329

Hereditary vitreoretinal dystrophy 195

Herpes simplex virus 157

virus keratitis 37

zoster 41 
- ophthalmicus 61 Herpetic keratitis 333 Histopathology 298 Human 82

parvovirus B 19161 Humidifier 216 Hypotony 318

Immune depression 49 Indocyanine green angiography 185 Infrared spectra 17 Inner limiting membrane 82 Interdisciplinary care 92 Intraocular lens 254,298

malignancies 119

pressure 32, 254, 309

347

Intrinsic sympathomimetic activity 32 Iridocorneal endothelial syndrome 289 Iris fluorescein angiography 172

- $\quad$ pigment epithelium 172

Jackson optotype 95

Kelman phacoemulsification procedure

131 Keratitis 157

Lens capsule 17 Levobunolol 32 Light exposure 220

microscopy 270

of video camera 145 Low-intensity background 136 Low-vision aids 92

training 92

Maculopathy 318

Magnetic resonance imaging 195,226 Mechanical properties 201 Medical therapy 333

Microfollicular conjunctivitis 295 Micro-Fourier transform infrared

spectrometry 17 MitomycinC 247 Moisture aid 216 Moll’s gland 54,267 Monoyer chart, geometrized 95 Mouse 220

Multifocal choroiditis 163 Multiple sclerosis 226 Mydriatic effect, decongestant 243 Myopia 163

Nasal cycle 110 Nd:YAG laser 122 Necrotic melanoma 65 Neuromyelitis optica 226

Neuroretinitis 168 Non-contact tonometry 115 Non-Hodgkin's lymphoma 65 Normal values 98

Normal-tension glaucoma 142 Normals 304

Occlusion 214 Octopus 1-2-3 10 Ocular hypertension 32

surgery 44 Oculotoxicity 98 Oncocytoma 54, 267 Operation technique 77 Optic disc cupping 336

nerve 230, 270

neuritis 110

Orbital metastasis 329 - vessels 304 Organ culture 1, 278

Papilloedema 230

Paranasal sinusitis 110

Paranoid hallucinations 44

Partially accommodative strabismus 71

Passive load 201

Pattern electroretinogram 259

- $\quad$ electroretinography 61Penetrating keratoplasty 105Perimetry 136

Phacoemulsification, aspiration 254 Photic maculopathy 145

Photo therapeutic keratectomy 198 Polyarteritis nodosa 168 Polygonal-type cell 267

Postcataract against-the-rule astigmatism 131

with-the-rule astigmatism 131 Posterior chamber 298

fixation sutures 71 Pressure 17

injury 270 Pressure-lowering effect 122 Program Ml 10 
M2X 10

Protective equipments 15 Proton relaxation times 195 Pseudoxanthoma elasticum 239

Psychiatric complications 44 Pterygium, recurrence 247 Pulsair tonometer 115 Pupillography

243

1 lq-syndrome 233

Rabbit 1

- $\quad$ eye 278

Radiolabeled precursor 278 Radiosurgery 119

Red-green color vision deficiency 136

Regeneration 270

Retina 82

Retinal diseases 163

pigment epithelium 29

venous collaterals 29

vessel 233 Retinitis pigmentosa 23

punctata albescens 339 Retinopathy 179

Rod mechanism 136

Sarcoidosis 230 Scleral buckling 250 Scopulariopsis brevicaulis 333 Sebaceous gland carcinoma 46

Secondary cataract 298

- $\quad$ malignancy 65Sensory thresholds 136Sequential enzymatic degradation 1Sex 142

Short TI inversion recovery 226 Snellen chart, regularized 95 Sodium iodate 220 Spleen cells

105 Sports-related eye injuries 15 Standardized echography 329 Stereotactic radiation 119

Steroids 321 Stickler's disease 195 Subretinal fibrosis 163 Suction attachment 119 Superoxide dismutase 270

Tcell 157

Thyroid-releasing hormone 270

Timolol maleate 207

Tonometer 309

Topical therapy 207

Topographical variation 82

Trabecular mesh work 1,278

Trabeculectomy 318

Transantral approach 284

Transconjunctival approach 284

Transmission electron microscopy 270

Trauma 15

Triazolam 145

Twofold-angle coordinates 250

Type IV collagen 17

Ultrastructure 82 Uncontrolled glaucoma 122 Uveal melanoma 119 Uveitis 163

Varicella-zoster virus DNA 41 Venous leakage 29 Viscoelastic materials 1 Visual acuity

standardization 95

field(s) 23, 142

prognosis 210

Visually evoked cortical potentials 259 
- $\quad$ - - response 61Vitreoretinal border region 82Vitreous body 195

Wet gauze eye mask 216

XPERT-CT tonometer 115

348

Subject Index 